\title{
A Novel Allele of Myosin VIIa Reveals a Critical Function for the C-Terminal FERM Domain for Melanosome Transport in Retinal Pigment Epithelial Cells
}

\author{
Martin Schwander, ${ }^{1}$ Vanda Lopes, ${ }^{2,3}$ Anna Sczaniecka, ${ }^{1}$ Daniel Gibbs, ${ }^{2}$ Concepcion Lillo, ${ }^{2}$ David Delano, ${ }^{4}$ \\ Lisa M. Tarantino, ${ }^{5}$ Tim Wiltshire, ${ }^{5}$ David S. Williams, ${ }^{2,3}$ and Ulrich Müller ${ }^{1}$ \\ ${ }^{1}$ Department of Cell Biology, Institute for Childhood and Neglected Disease, The Scripps Research Institute, La Jolla, California 92037, ${ }^{2}$ Departments of \\ Pharmacology and Neuroscience, School of Medicine, University of California at San Diego, La Jolla, California 92093, ${ }^{3}$ Departments of Ophthalmology and \\ Neurobiology, Jules Stein Eye Institute, School of Medicine, University of California Los Angeles, Los Angeles, California 90095, ${ }^{4} \mathrm{Genomics}$ Institute of the \\ Novartis Research Foundation, San Diego, California 92121, and 5Department of Psychiatry, University of North Carolina, Chapel Hill, North Carolina 27516
}

Mutations in the head and tail domains of the motor protein myosin VIIA (MYO7A) cause deaf-blindness (Usher syndrome type 1B, USH1B) and nonsyndromic deafness (DFNB2, DFNA11). The head domain binds to F-actin and serves as the MY07A motor domain, but little is known about the function of the tail domain. In a genetic screen, we have identified polka mice, which carry a mutation (c.5742+ 5G $>$ A) that affects splicing of the MY07A transcript and truncates the MY07A tail domain at the C-terminal FERM domain. In the inner ear, expression of the truncated MY07A protein is severely reduced, leading to defects in hair cell development. In retinal pigment epithelial (RPE) cells, the truncated MY07A protein is expressed at comparative levels to wild-type protein but fails to associate with and transport melanosomes. We conclude that the C-terminal FERM domain of MYO7A is critical for melanosome transport in RPE cells. Our findings also suggest that $M Y O 7 A$ mutations can lead to tissue-specific effects on protein levels, which may explain why some mutations in MYO7A lead to deafness without retinal impairment.

\section{Introduction}

MYO7A consists of an N-terminal motor domain followed by a neck and tail domain (Chen et al., 1996). The motor domain enables movement of MYO7A on actin filaments. The tail domain interacts with vesicle-associated proteins such as Slac2-c/MyRIP suggesting that MYO7A might play a role in cargo transport (Kuroda and Fukuda, 2005; Soni et al., 2005; Klomp et al., 2007). Over 100 mutations have been identified in MYO7A that affect the head and tail domains and cause syndromic (USH1B) and nonsyndromic (DFNB2, DFNA11) deafness (http://www.hgmd.cf.ac.uk/ac/gene.php?gene=MYO7A). Mutations in the head domain are thought to affect MYO7A motor function, but little is known about the mechanisms by which mutations in the tail domain affect protein function.

Received Sept. 30, 2009; accepted 0ct. 30, 2009.

This work was funded by National Institutes of Health (NIH) Grants DC005965 and DC007704 (U.M.), NIH Grant EY07042 and Core Grant EEY00331 (D.S.W.), the Skaggs Institute for Chemical Biology (U.M.), and a fellowship from the Bruce Ford and Anne Smith Bundy Foundation (M.S.). D.S.W. is a Jules and Doris Stein Research to Prevent Blindness Professor. We thank members of the Müller laboratory for helpful discussions, and Tama Hasson for MY07A antibodies.

D. Gibbs's present address: The Salk Institute for Biological Studies, 10010 North Torrey Pines Road, La Jolla, CA 92037.

C. Lillo's present address: Instituto de Neurociencias de Castilla Leon, Universidad de Salamanca, Salamanca 37007, Spain.

Correspondence should be addressed to Ulrich Müller, The Scripps Research Institute, 10550 North Torrey Pines Road, La Jolla, CA 92073. E-mail: umueller@scripps.edu.

D01:10.1523/JNEUROSCI.4876-09.2009

Copyright $\odot 2009$ Society for Neuroscience 0270-6474/09/2915810-09\$15.00/0
Studies in mice have provided insights into the cellular mechanisms by which mutations in MYO7A cause disease. Myo7a mutations in shaker-1 mice cause recessive deafness, vestibular dysfunction, and retinal abnormalities (Gibson et al., 1995; Liu et al., 1997). In the inner ear, MYO7A is expressed in mechanosensory hair cells and is required for hair bundle morphogenesis and mechanotransduction (Self et al., 1998; Kros et al., 2002). Within the retina, MYO7A localizes to the cilium of the photoreceptors, to the apical region of retinal pigment epithelial (RPE) cells, and to melanosomes within RPE cells (Wolfrum et al., 1998; ElAmraoui et al., 2002; Gibbs et al., 2004). In accordance with its expression pattern, MYO7A regulates opsin transport in photoreceptors and the phagocytosis of shed outer segments by RPEs (Liu et al., 1999; Gibbs et al., 2003). Melanosomes fail to localize to the apical processes in RPEs of shaker-1 mice, indicating that MYO7A is required for movement and/or retention of melanosomes within the apical processes (Liu et al., 1998). Unlike the human patients, shaker-1 mice do not show degeneration of photoreceptor cells, suggesting that the mice mimic only some aspects of the human disease. Nevertheless, mice provide currently the best available animal model for the human disease.

Here, we report a novel Myo7a allele termed polka that we isolated in a genetic screen in mice (Schwander et al., 2007). Polka mice carry a point mutation (c.5742 $+5 \mathrm{G}>\mathrm{A}$ ) in intron 42 that affects splicing and is predicted to truncate the tail domain of MYO7A at its C-terminal FERM domain. While the mutant MYO7A protein was expressed in the retina, little expression was 


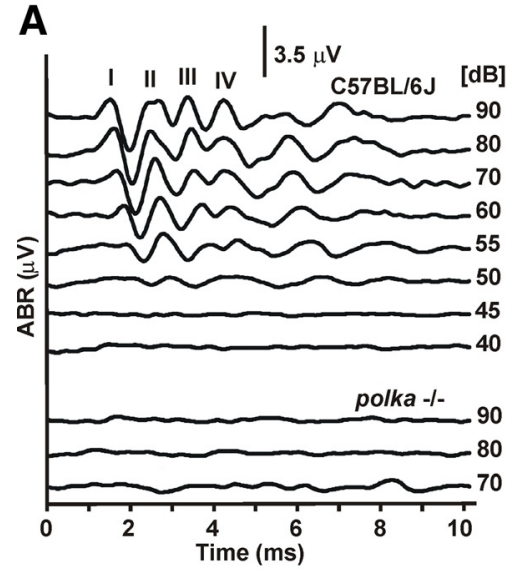

B

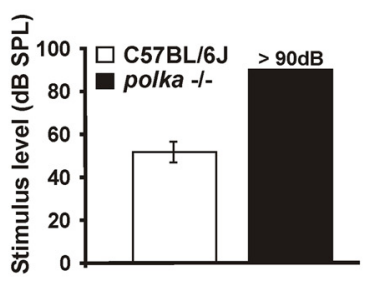

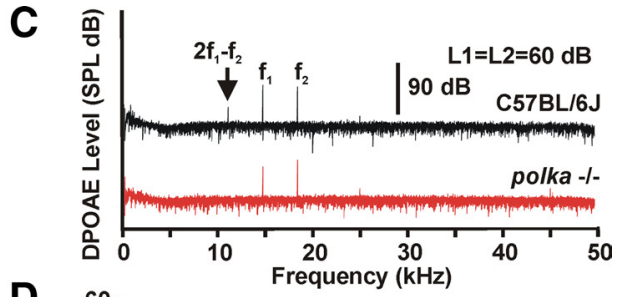
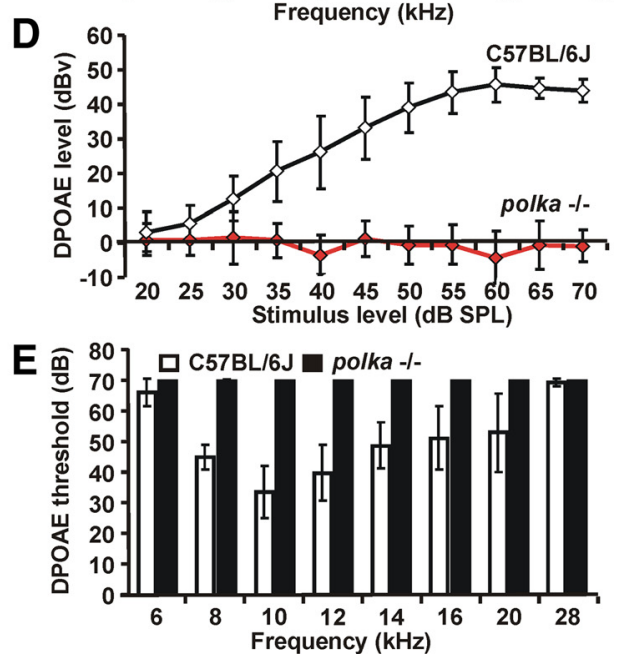

Figure 1. Auditory function in polka mice. $A$, Auditory brainstem response (ABR). Representative examples of click-evoked ABR waveforms for a $(57 \mathrm{BL} / 6 \mathrm{~J}$ wild-type and a polka homozygous mutant mouse at different sound intensities (in decibels). ABR waves I-IV are indicated for recordings obtained from a wild-type mouse. The polka mutant mouse failed to respond to all tested sound intensities (0-90 dB). $\boldsymbol{B}$, Average auditory thresholds for 2-month-old mice (wild type $n=4$, polka $n=9$; the mean \pm SD). C, Representative DPOAE response spectra for a wild-type (top trace) and a polka mouse (bottom trace) at a single stimulus condition (median primary frequency $=16 \mathrm{kHz}$ ). Note the cubic distortion product $\left(2 f_{1}-f_{2}\right)$, which is absent in recordings with polka mice at $60 \mathrm{~dB}$. D. Cubic distortion product levels as a function of stimulus intensity at $10 \mathrm{kHz}$ from wild-type and polka mice. No response was observed in polka mice at all intensity levels (wild type $n=4$, polka $n=9$; mean \pm SD). $\boldsymbol{E}$, DPOAE thresholds were highly elevated at all frequencies analyzed (wild type $n=4$, polkan $=9$; mean \pm SD). Primary frequencies were maintained at an $f_{2} / f_{1}$ ratio of 1.22 , and $L_{1}$ was equal to $L_{2}$.

observed in the inner ear. Hair bundles in the inner ear showed morphological defects that likely caused the deafness phenotype of polka mice. In the retina, the mutant MYO7A protein was still localized to the apical processes of RPE cells, but associated less well with melanosomes, which were mislocalized. We conclude that the C-terminal FERM domain of MYO7A is important for association with and transport of melanosomes. In addition, our findings show that a point mutation in a gene can differentially affect its expression in the inner ear and retina, a finding that might be relevant to understanding disease mechanisms associated with mutations in $M y o 7 a$.

\section{Materials and Methods}

ENU mutagenesis screen and evaluation of inner ear function. The ENU mutagenesis protocol and primary phenotypic screen has been described previously (Reijmers et al., 2006; Schwander et al., 2007). The measurement of auditory brainstem responses (ABRs), distortion product otoacoustic emissions (DPOAEs), and vestibular function followed our published procedures (Schwander et al., 2007).

Linkage analysis and DNA sequencing. Linkage analysis using single nucleotide polymorphism (SNP) markers was performed as described previously (Wiltshire et al., 2003; Schwander et al., 2007). Affected polka mice were bred with $129 \mathrm{~S} 1 / \mathrm{S} v \mathrm{ImJ}$ mice. The $\mathrm{F}_{1}$ offspring were intercrossed to obtain $\mathrm{F}_{2}$ mice and tail DNA was prepared for linkage mapping using SNPs markers (Wiltshire et al., 2003). Map Manager QTX (Manly et al., 2001) was used to calculate logarithm of the odds (LOD) scores and perform interval mapping. Exons and exon-intron boundaries of genes in the mapped intervals were sequenced. Primers for PCR amplification and DNA sequencing were designed with Primer 3 software (MIT). DNA numbering is based on Myo7a cDNA (NM_008663) and starts with +1 as the A of the ATG initiation methionine.

Histology, immunohistochemistry, and scanning electron microscopy. Staining of sections and TUNEL staining were performed as described previously (Fariñas et al., 1996; Müller et al., 1997; Gibbs et al., 2004). Whole-mount staining and scanning electron microscopy of cochlear sensory epithelia were performed as described previously (Senften et al., 2006; Schwander et al., 2007).

Immunoelectron microscopy and gold particle quantitation. For immunoelectron microscopy, eyecups were fixed by immersion in $0.25 \%$ glutaraldehyde, $4 \%$ paraformaldehyde in $0.1 \mathrm{M} \mathrm{ca}$ codylate buffer, pH 7.4. Samples were embedded in LR White (EMS). Ultrathin sections $(70 \mu \mathrm{m})$ were etched with saturated sodium periodate and blocked with $4 \%$ bovine serum albumin (BSA) in antibody buffer $(1 \%$ $\mathrm{BSA}+1 \%$ Tween 20) for $1 \mathrm{~h}$. The sections were then incubated with primary antibodies (antiMYO7A and anti-MYRIP) overnight at $4^{\circ} \mathrm{C}$. After washing, samples were incubated with goat anti-rabbit IgG conjugated to $12 \mathrm{~nm}$ gold (EMS) for $1 \mathrm{~h}$. Finally sections were stained with uranyl acetate and lead citrate. Sections that were not incubated with the primary antibody or shaker-1 sections (sh $1^{4626 S B}$ ) (Rinchik and Carpenter, 1999) were processed at the same time and used as negative controls. Immunolabeling density was determined by counting gold particles in ultrathin sections. Images were taken randomly along the RPE, 25 per condition, and analyzed. Gold particles were considered to be associated with the melanosome membrane when located at a maximum of $30 \mathrm{~nm}$ of the membrane of the organelle, as described previously (Klomp et al., 2007). Section area was determined with ImageJ software.

Complementation tests and genotyping. Heterozygous polka mice were crossed with homozygous sh1 $1^{4626 S B}$ mice, which carry a predicted Myo7a-null allele (Mburu et al., 1997; Rinchik and Carpenter, 1999). Auditory thresholds were determined by ABR tests. Genotyping was performed by PCR using a set of primers that flank the polka mutation in the $M y o 7 a$ gene: forward primer, 535f 5' -GGTCTTGCAGAAGTTGAGTG$3^{\prime}$, and reverse primer 535r 5'-AAGCTTTGCTGCCATGTACC-3'. PCR fragments were purified and digested with BstYI to give a $150 \mathrm{bp}$ product in homozygous mutants, and $300 \mathrm{bp}$ and $150 \mathrm{bp}$ products in heterozygous littermates. For the Myo $7 a^{4626 S B}$ mutation genotyping was performed as described previously (Holme and Steel, 2002).

RT-PCR and quantitative PCR. RNA was isolated from cochleas and eyes by using Trizol (Invitrogen), according to the manufacturer's instructions. RNA concentration was determined using Nanodrop. cDNA was synthesized from $400 \mathrm{ng}$ of RNA with Superscript III reverse transcriptase (Invitrogen) and oligo(dT) primers. RT-PCR analysis for the splicing of Myo7a transcripts was performed with primers exon41f 5'-CATAAGACTACCCAGATCTTC-3', exon42f 5' -GGCTGCTGCTCAAGTCTTC-3', exon42r 5'-GAAGACTTGAGCAGCAGCC-3', and exon43r 5'-GAAATCATTCTCTGGGACGC-3'. Gene expression was assessed by quantitative PCR by using gene-specific primers and SYBR green (Applied Biosystems) in a PTC-200 thermal cycler (Bio-Rad) coupled to a Chromo4 real-time PCR detection system (Bio-Rad). Myo7a mRNA expression data were normalized by using cadherin 23 (Cdh23) and otoferlin (Otof), as well as the housekeeping genes $36 B 4$ and GAPDH, as reference genes. The primer sequences recognizing $M y o 7 a$ were as follows: forward primer, myo7a_5431f1 5' -ATCCTCCTGCCTCATGTTCAG-3', reverse primer, myo7a_5594r1 5' -CGGGGAAGTAGACCTT- 
GTGGA-3'; for cadherin 23: forward primer, cdh23_6157f3 5'-GCCCACCTGTTCATCACTATC-3', reverse primer, cdh23_6260r3 5'-TGGCTGTGACTTGAAGGACTG-3'; for otoferlin: forward primer, otof_4059f3 $5^{\prime}$ GGAAGAGAAGGAAGAGATGGAAAG-3', reverse primer, otof_4143r3 5'-GGGCTCTGGTTTTTCTTCTTTTTC-3'.

\section{Results}

Analysis of auditory and vestibular function in polka mice

We have previously described a forward genetics screen in mice aimed at identifying recessive deafness traits (Schwander et al., 2007). One of the lines from the screen, termed polka, showed prominent circling behavior and performed poorly in forced swim tests, indicative of vestibular dysfunction. Polka mice also failed to show an acoustic startle response (ASR) (Schwander et al., 2007). As defects in the ASR can be caused by auditory defects, general defects in the nervous system or altered motor function, we next tested polka mice for auditory function by evaluating their ABR (Zheng et al., 1999). To determine auditory thresholds we applied broadband click stimuli to 2-month-old mice starting at $90 \mathrm{~dB}$ and then decreasing in intensity. In polka mice ABR thresholds were highly elevated ( $>90 \mathrm{~dB}$ ) when compared to wild-type C57BL/6J mice (Fig. $1 A, B)$, suggesting that the auditory phenotype can be attributed to impaired hair cell or neuronal function. To study hair cell function, we measured the DPOAEs. In wild-type mice, DPOAEs were dependent on the stimulus intensity at a given frequency, but were not detectable in the mutants, as shown in a plot of DPOAE level versus stimulus level at the mean primary frequency of $10 \mathrm{kHz}$ (Fig. 1C, D). Similar observations were made at all of the frequencies analyzed $(6-28 \mathrm{kHz})$ (Fig. $1 E)$, indicating that the function of outer hair cells was impaired across the entire analyzed frequency spectrum.

\section{Polka is a novel allele of the Myo7a gene}

As part of the original genetic screen we performed heritability testing and demonstrated that polka mice, which were derived on a C57BL/6J background, inherit their deafness/balance phenotype recessively (Schwander et al., 2007). To map and positionally clone the affected gene, we outcrossed affected polka mice to $129 \mathrm{~S} 1 /$ SvImJ mice. The resulting offspring was intercrossed to obtain $\mathrm{F}_{2}$ mice for $\mathrm{ABR}$ phenotyping, tail DNA preparation, and SNP mapping. Consistent with a nonlethal recessive trait, we found $\sim 21 \%$ of $190 \mathrm{~F}_{2}$ animals analyzed to be affected (Schwander et al., 2007).

Using tail DNA from nine affected and four unaffected $\mathrm{F}_{2}$ animals (26 meiotic events), we ran SNP arrays as previously described (Wiltshire et al., 2003; Schwander et al., 2007). The mutation in polka mice mapped to a $27 \mathrm{MB}$ interval on chromo-

B

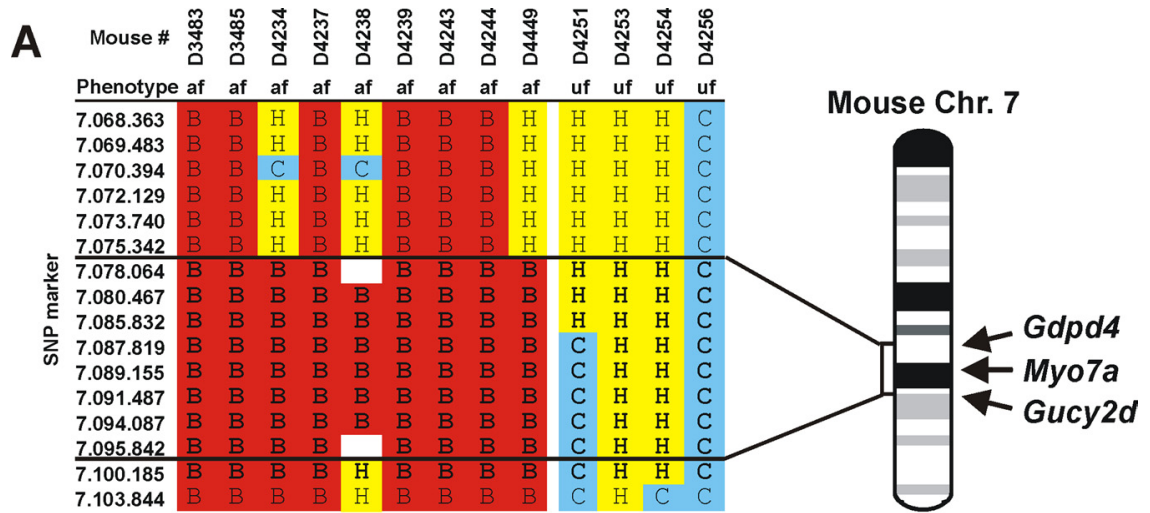

Figure 2. SNP mapping of the mutation in polka mice. A, DNA from nine affected mice (af) and four unaffected mice (uf) was analyzed for SNP markers on chromosome 7, which are listed in the first column and indicate the megabase position

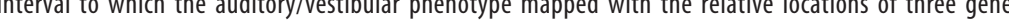
mice at the $3^{\prime}$-end of exon 42 of Myo7a. A G-to-A transition $(c .5742+5 G>A)$ that was found only in affected mice is boxed. The point mutation creates a BstYI restriction site, facilitating genotyping of the mice. $C$, An agarose gel picture shows the resolved fragments from BstYI RFLP/PCR analysis on genomic DNA of a wild-type, and a homozygous polka heterozygous $\operatorname{sh} 1^{46265 B}$ mice. Note that in the offspring only mice that were compound heterozygotes for the polka and $s h 1^{4626 S B}$ alleles showed elevated auditory thresholds.

some 7 (Fig. 2 A). We next sequenced the exons and exon-intron boundaries of all annotated and predicted genes in the interval, including the Myo7a gene, which has previously been linked to deafness in mice and humans (Gibson et al., 1995; Weil et al., 1995). Sequencing of all exons of Myo7a from multiple affected and unaffected control mice did not reveal any point mutations. However, a G-to-A transition (c.5742 + 5G > A, reference sequence: NM_008663) was present at the fifth position of intron 42 , and uniquely homozygous in mice that displayed the deafness phenotype (Fig. 2B) (112 mice analyzed). The point mutation was confirmed by restriction analysis (Fig. $2 B, C$ ). No mutation was found in any other gene in the interval (data not shown).

To confirm that the point mutation in the Myo7a gene caused the deafness phenotype, we performed complementation tests with shaker-1 mice (sh $1^{4626 S B}$ ) (Fig. 2D) (Rinchik et al., 1990; Rinchik and Carpenter, 1999). The Myo7 $a^{4626 S B}$ mutation intro- 
A

Predicted Donor Splice Site Motif TAGGTGG[G/A]T $\mathrm{ATGccc}$

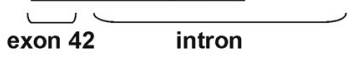

Splice score:

G (wild-type): 88

A (mutant): 82

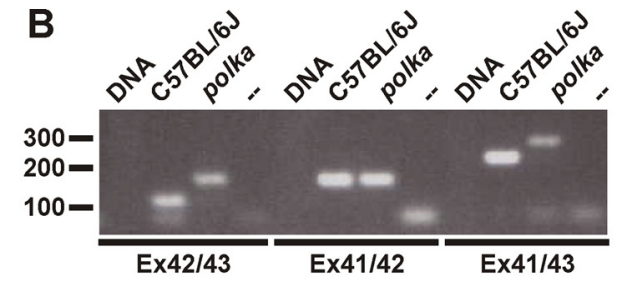

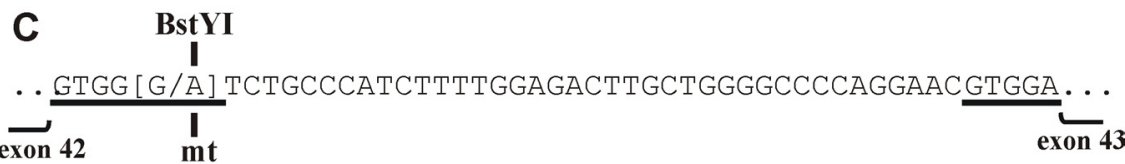

D MYO7A

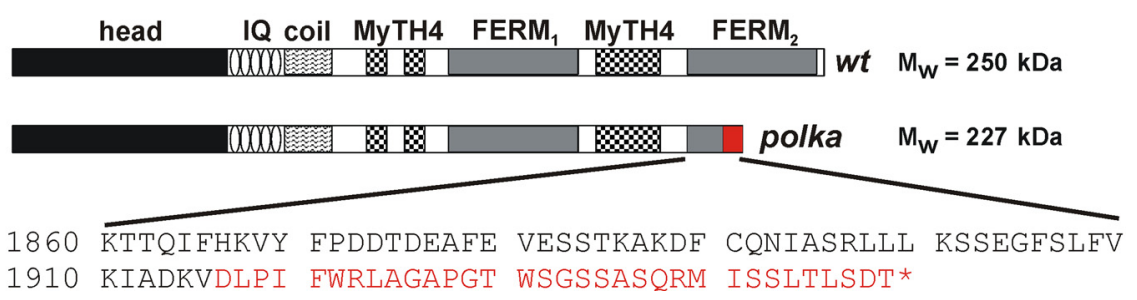

Figure 3. RT-PCR analysis of mRNA from the Myo7a locus. $A$, Predicted donor splice site motif and splice score. $B, R T-P C R$ analysis of the Myo7a transcripts obtained from the inner ear (P5) from wild-type and polka mice. PCR analysis of genomic DNA from wild-type mice was included as a control. Note that fragments including the polka mutation (Ex42/43, Ex41/43) were $\sim 50$ bp larger than fragments amplified from wild-type mRNA. C, Sequence determination of Myo7a PCR products generated from mutant mice. A splicing defect induced the retention of the first $49 \mathrm{bp}$ of intron 42. D, Domain structure of MY07A. The polka mutation creates a frameshift and introduces a premature stop codon, which is predicted to truncate the protein within the $\mathrm{FERM}_{2}$ domain.

duces a stop codon near the $5^{\prime}$ end, which likely results in a functional null allele (Hasson et al., 1997; Mburu et al., 1997). We crossed homozygous polka mice with heterozygous sh $1^{4626 S B}$ mice, genotyped the offspring, and determined auditory thresholds by measuring ABRs. Mice that were compound heterozygotes for the polka and sh1 $1^{4626 S B}$ alleles tested deaf, while littermates carrying one polka allele and one wild-type allele showed normal auditory thresholds (Fig. 2D). Heterozygous sh $1^{4626 S B}$ mice also had normal hearing function (data not shown). We conclude that the point mutation in the Myo7a gene is responsible for the deafness phenotype in polka mice.

The polka mutation affects splicing of the Myo7a transcript The G-to-A transition in Myo7a disrupts the $3^{\prime}$ end of the U1 snRNP binding site (Fig. 3A) (Blencowe, 2000). Applying the statistical Shapiro-Senapathy splicing algorithm (Senapathy et al., 1990), splice scores of 0.82 and 0.88 result for the mutant and the wild-type variant, respectively. The lower splice score indicates a lowered binding affinity of the mutated splice site with the corresponding U1 snRNP, which could result in either exon skipping or cryptic splice site activation (Krawczak et al., 2007). To examine the effect of the mutation on the splicing of Myo7a transcripts, we performed reverse-transcription PCR (RT-PCR) amplification on total RNA isolated from vestibular sensory patches from wild-type and polka mice. Amplification with primers targeting exon 42 and 43 , which are located $5^{\prime}$ and $3^{\prime}$ of the mutated intron 42, identified a novel Myo7a mRNA species in mutant samples, which was $\sim 50$ bp larger than the wild-type transcript (Fig. $3 B$ ). Subcloning and DNA sequencing of the amplified products revealed a $49 \mathrm{bp}$ insert between exons 42 and 43 in polka mice (Fig. $3 C$ ). The sequence of the insert completely matched part of intron 42 indicating that the mutation in polka mice led to the utilization of a cryptic splice site in intron 42 (GA/GTGGGT) generating an altered transcript (Myo7a42 + 49) that includes intronic sequences. The MYO7A C-terminal tail contains two FERM domains (Fig. 3D) (Chen et al., 1996). Based on our sequencing data we predicted that the polka mutation leads to premature truncation of MYO7A after the first 56 aa of the C-terminal FERM domain (referred to in the following as $\mathrm{FERM}_{2}$ ) and the addition of a 33-aa-long aberrant C-terminal peptide (Fig. 3D).

Defective hair bundle morphology and melanosome localization in polka mice Myo7a mutations in shaker-1 mice lead to defects in hair bundle development in the inner ear and to defects in the localization of melanosomes to the apical process of RPE (Liu et al., 1998; Self et al., 1998). To determine whether the polka mutation caused similar phenotypic defects, we first stained cochlear sensory epithelia from heterozygous and homozygous polka mice as whole mounts with phalloidin to label F-actin in stereocilia (Fig. 4). In polka mice, stereociliary bundles were disorganized in all four rows of hair cells. Fragmented bundles with defective morphology were present that contained a small number of short stereocilia (Fig. $4 A, B$, arrows), and some bundles failed to develop a clear polarity in the apical hair cell surface (Fig. $4 B$, asterisk). These findings were confirmed by scanning electron microscopy (Fig. 4C-H). Bundles were smaller, many of the stereocilia were short and malformed (Fig. $4 D, H$, arrows), and polarity defects were evident (Fig. $4 D, H$, asterisk).

Next we analyzed retinal sections from polka mice (Fig. 5). At the light microscopic level, 2-month-old polka mice revealed no obvious structural defects in the retina, including the photoreceptors. However, unlike in wild-type mice, melanosomes did not localize to the apical processes of RPE cells (Fig. 5B, arrowheads). We conclude that the polka mutation leads to similar phenotypic manifestations in the ear and retina as observed in shaker-1 mice.

\section{MYO7A expression is affected in a tissue-specific manner}

Several shaker-1 mutations map to the myosin motor domain and are thought to affect both motor function and MYO7A protein levels (Hasson et al., 1997; Mburu et al., 1997). As the mutation in polka mice maps to the tail domain, we hypothesized that it might affect hair cells and RPE cells by a different mechanism. As one possibility, truncation of the FERM $\mathrm{F}_{2}$ domain might affect protein function in cargo transport. However, the mutation might also lead to instability of the RNA or protein thereby affecting MYO7A levels. To distinguish between these possibilities, we compared the expression of the MYO7A transcript and protein in wild-type and polka mice.

Murine MYO7A is a $250 \mathrm{kDa}$ polypeptide that is highly expressed in the cochlea, retina, testis, lung, and kidney (Hasson et al., 1995). To analyze MYO7A protein expression, we performed Western blots on tissue extracts from wild-type and mutant mice 


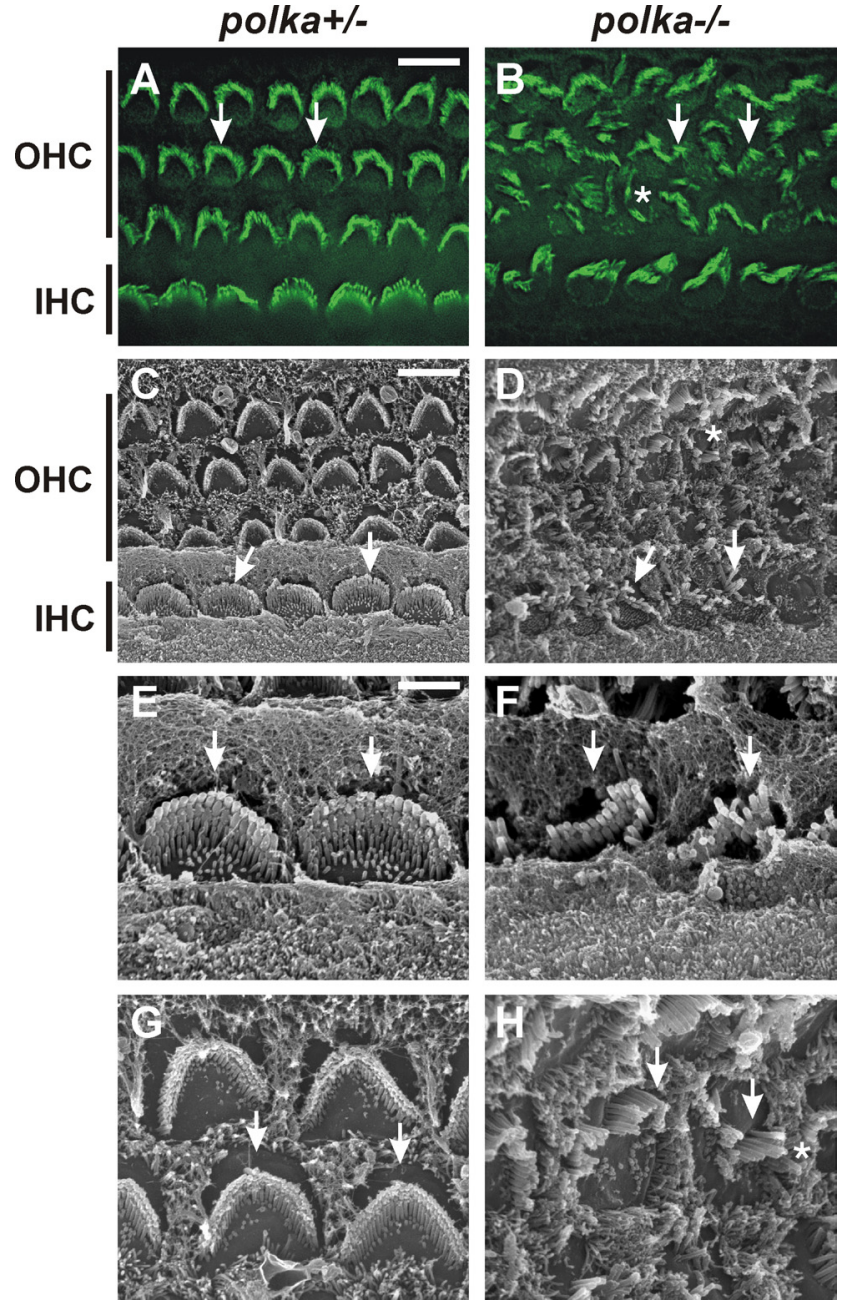

Figure 4. Analysis of hair bundle morphology. $A, B$, Images of phalloidin-stained whole mounts from the middle turn of the cochlea of heterozygous $(\boldsymbol{A})$ and homozygous $(\boldsymbol{B})$ polka mice at postnatal day 5 (P5). In polka mice morphological changes were evident such as splaying of stereocilia (arrows) and cell polarity defects (asterisk). $\mathbf{C} \boldsymbol{H}$, Scanning electron micrographs of cochlear whole mounts of $P 5$ heterozygous $(\boldsymbol{C}, \boldsymbol{E}, \boldsymbol{G})$ and homozygous $(\boldsymbol{D}, \boldsymbol{F}, \boldsymbol{H})$ polka mice. Note that hair bundles were smaller and fragmented in homozygous mutants (arrows). Scale bars: $\boldsymbol{A}, \boldsymbol{B}, 8 \mu \mathrm{m} ; \boldsymbol{C}, \boldsymbol{D}, 5 \mu \mathrm{m} ; \boldsymbol{E}-\boldsymbol{H}, 2 \mu \mathrm{m}$.

(Fig. 6A). A $250 \mathrm{kDa}$ protein was detected in extracts from brain, kidney, eye and ear in wild-type mice. In eye and brain extracts of homozygous mutant mice, the band for MYO7A was of similar intensity as in wild type, but it was shifted to a slightly smaller size. In eye extracts from heterozygous mutants two bands of the predicted size of full-length and mutant MYO7A could be distinguished (Fig. 6B). These findings support the interpretation of our sequencing results, and indicate that as a consequence of aberrant splicing a truncated MYO7A protein is expressed in the brain and eye of polka mice. Quantification of expression levels using Western blots from eye extracts confirmed that MYO7A expression levels in the retina were not reduced (Fig. 6C). In contrast, MYO7A expression levels were strongly reduced in extracts from mutant kidney and cochlea (Fig. 6A, C). We conclude that the polka mutation leads to tissue-specific effects in the expression of MYO7A.

To further define the mechanisms that caused tissue-dependent instability of mutant MYO7A, we compared mRNA levels of $M y o 7 a$ in eye and ear tissues from polka and wild-type mice by quantitative RT-PCR analysis. Expression of Myo7a transcripts

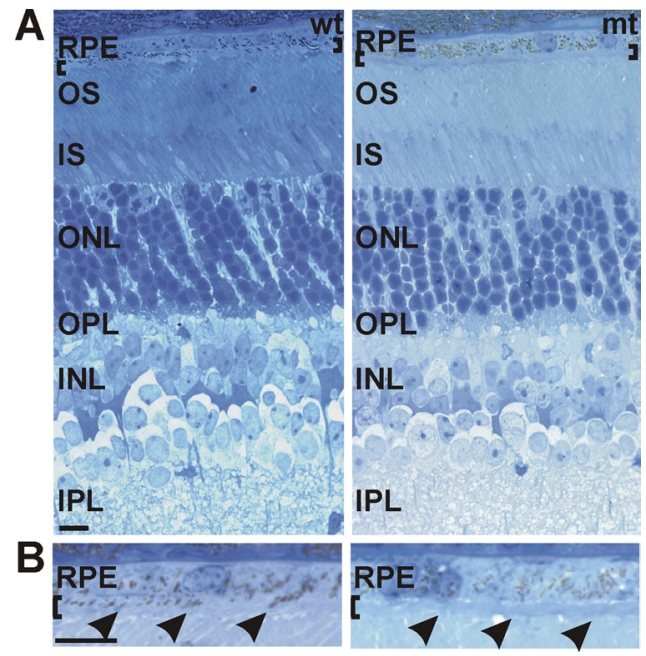

Figure 5. Defects in melanosome localization. $\boldsymbol{A}, \boldsymbol{B}$, Retinal semithin sections from 6-month-old mice. Polka mice showed no signs of retinal degeneration $(\boldsymbol{A})$. Melanosomes failed to localize in apical processes of mutant RPE cells ( $\boldsymbol{B}$, arrowheads). OS, Photoreceptor outer segments; IS, photoreceptor inner segments; ONL, outer nuclear layer; OPL, outer plexiform layer; INL, inner nuclear layer; IPL, inner plexiform layer; Scale bars: $\boldsymbol{A}, \boldsymbol{B}$, $10 \mu \mathrm{m}$.

\section{A}

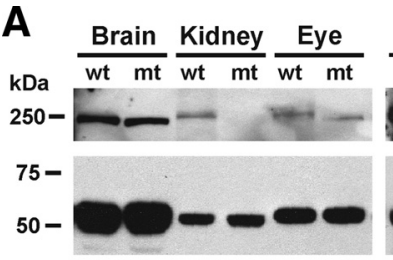

C

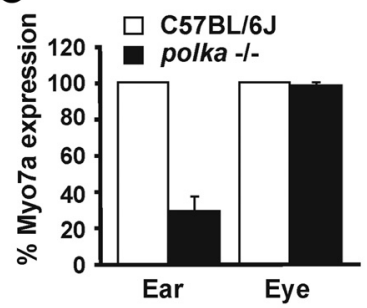

$\mathbf{E}$

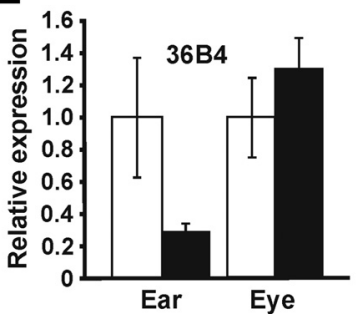

B
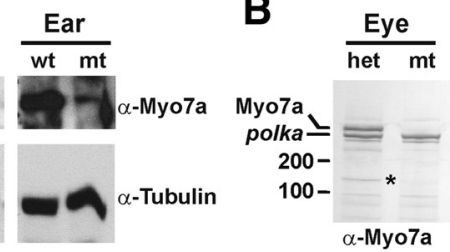

D

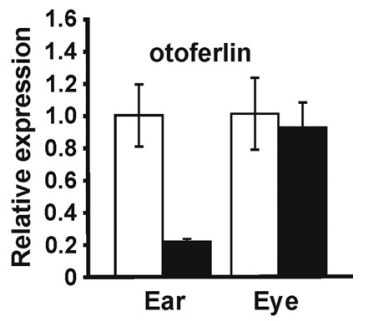

F

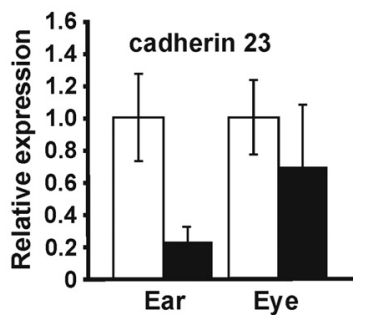

Figure 6. MY07A protein and mRNA expression. $\boldsymbol{A}$, Western blot analysis revealed a slightly reduced molecular weight for MY07A in brain and eye extracts from mutant mice, while its expression was drastically reduced in kidney and cochlea. $\boldsymbol{B}$, Wild-type and mutant MY07A were detected as a double band in eye extracts from heterozygous polka mice. An additional smaller band of unknown origin was identified only in extracts from heterozygous mice (asterisk). C, Densitometry of Western blots. $\boldsymbol{D}-\boldsymbol{F}$, Real-time RT-PCR analysis. mRNA levels of Myo7a in ear and eye were quantified in relation to otoferlin (0tof) (D), 36B4 (E), and cadherin $23(\mathrm{Cdh} 23)(\boldsymbol{F})$. Levels of Myo7a were drastically decreased in ears, but not in eyes of polka mice. Data are expressed relative to levels of Myo7a transcripts in wild type [set as equal to 1; mean \pm SEM of 4 animals ( polka) and 3 animals (wild type) per group]. 

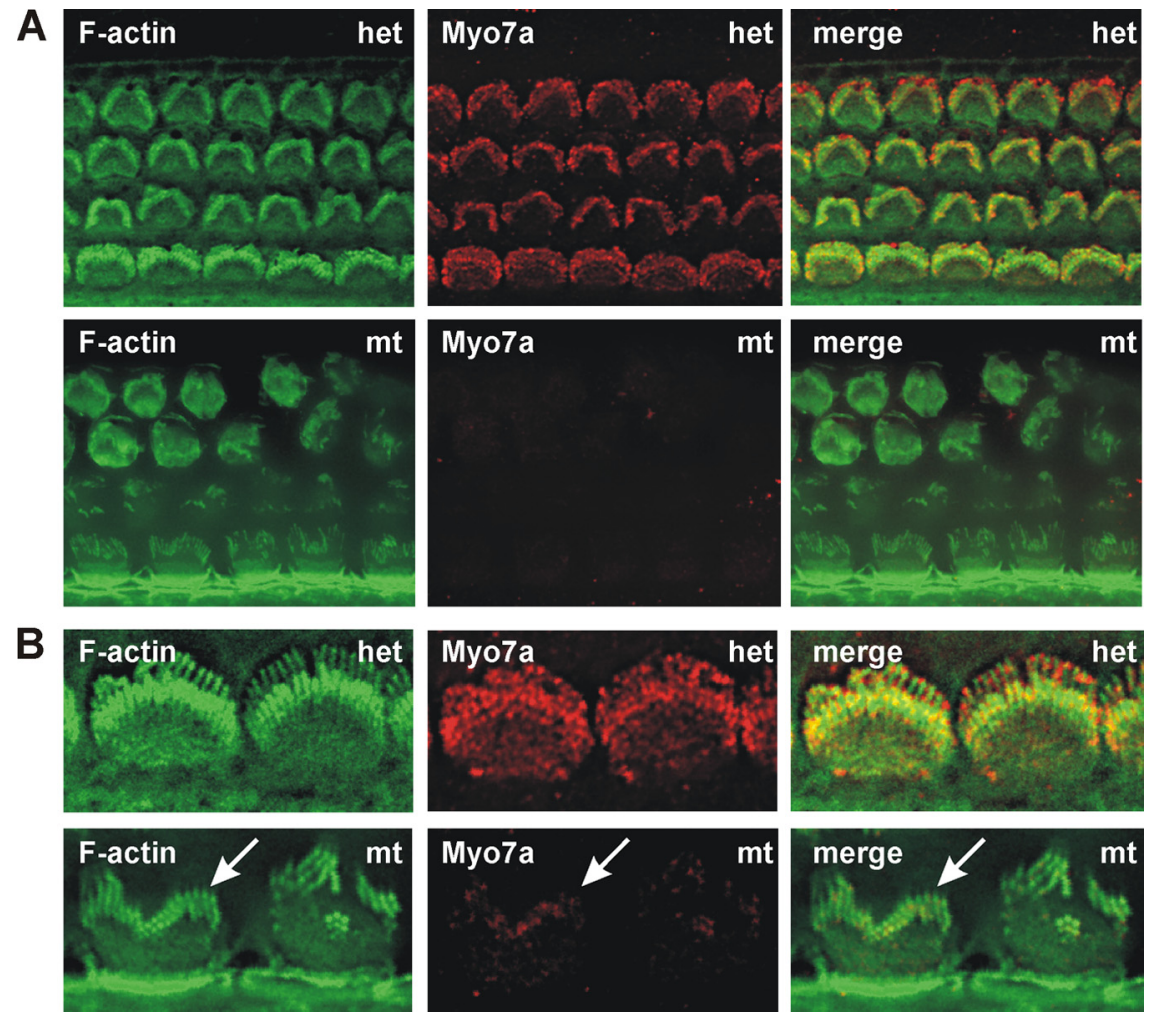

mt

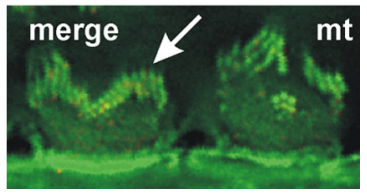

Figure 7. MY07A expression in hair cells. A, Cochlear whole mounts of heterozygous and homozygous polka mice at P5 were stained with phalloidin (green) to reveal hair cell stereocilia and an antibody against MY07A (red). MY07A expression was detected in outer and inner hair cells of heterozygous mice but was absent in the mutants. $\boldsymbol{B}$, At higher magnification, MY07A was localized to the cuticular plate and toward tips of stereocilia in heterozygous mice. In mutant hair cells, MY07A could no longer be detected. Occasionally, very low amounts of protein could be visualized at tips of stereocilia in some hair cells (arrows). Scale bars: $A, B, 8 \mu \mathrm{m}$.

Interestingly, while reduced levels of a truncated MYO7A protein were still expressed in the inner ear of polka mice, the protein was no longer detectable in stereocilia (Fig. $7 A, B$ ), suggesting that the residual protein was confined to the cell body. We next determined the expression and localization of the truncated MYO7A protein in the retina of polka mice by immunohistochemistry. At the light microscopic level, MYO7A was still localized to the apical processes of RPE cells, although the signal appeared more diffuse and was more widespread (Fig. 8A,B), indicative of changes of MYO7A distribution within RPE cells.

Previous studies have provided evidence that the exophilin, Slac2-c/MYRIP, binds to a domain in MYO7A that includes the $\mathrm{FERM}_{2}$ domain, which is affected by the polka mutation. Furthermore, Slac2-c/ MYRIP links RAB27A on melanosomes to MYO7A (El-Amraoui et al., 2002; Fukuda and Kuroda, 2002; Kuroda and Fukuda, 2005; Klomp et al., 2007). The functional significance of these interactions has remained unclear. We hypothesized that these interactions might be important for melanosome transport into the apical processes of RPE cells, and that this process might be affected in polka mice. We therefore determined the subcellular distribution of MYO7A and Slac2-c/MYRIP in
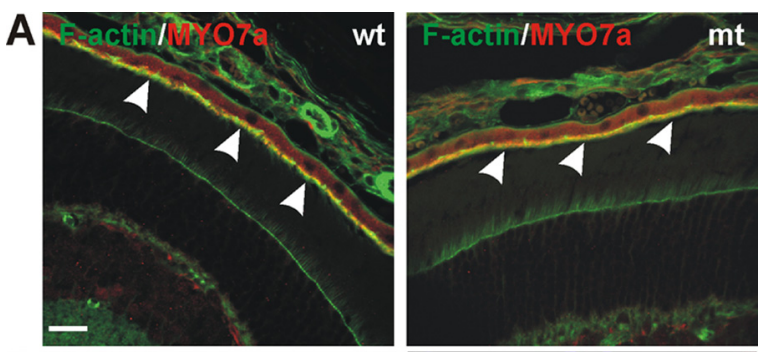

$\mathrm{B}$
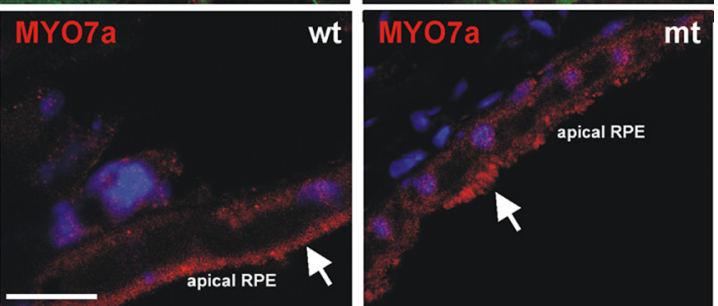

Figure 8. MY07A expression in the retina. $\boldsymbol{A}, \boldsymbol{B}$, Retinal cryosections from wild-type and polka mutant mice were stained with antibodies against MY07A (red) and with phalloidin (green) to visualize F-actin ( $\boldsymbol{A})$. In $\boldsymbol{B}$, high-magnification views of stainings for MY07A (red) are shown. Expression of MY07A in the apical RPE of polka mice was still detectable but not as well confined to the apical region of the cells compared to wild type ( $\boldsymbol{B}$, arrows). Scale bars: $A, B, 10 \mu \mathrm{m}$.

was strongly decreased in ear tissue of mutant mice when compared with wild type (Fig. $6 D-F$ ). In contrast, no significant difference in the levels of Myo7a was observed in the eye (Fig. $6 D-F)$, suggesting that mutant mRNAs in the ear but not the retina are degraded by the nonsense-mediated decay pathway (Isken and Maquat, 2008).
RPEs by immunoelectron microscopy (Fig. 9). Quantification of immunogold showed that total levels of MYO7A and MYRIP were unaffected in RPE cells and in photoreceptor cilia of mutant mice (Fig. 9; supplemental Fig. 1, available at www.jneurosci.org as supplemental material; and data not shown), consistent with the Western blot data. However, the fraction of MYO7A associated with melanosomes was significantly reduced in mutants compared to wild type, and the number of melanosomes without MYO7A was significantly increased. In parallel, MYO7A levels in the apical region of RPE cells were reduced, and in the basal region increased, indicating that MYO7A was redistributed in the mutants. The data suggest that the $\mathrm{FERM}_{2}$ domain is required for the recruitment of MYO7A to melanosomes (Fig. 9A, $B, E$ ). In contrast, the density of Slac2-c/MYRIP on melanosomes was unaffected (Fig. 9C-E). As Slac2-c/MYRIP also binds to the melanosome associated protein RAB27A, our findings suggest that Slac2-c/MYRIP is recruited independently of MYO7A to melanosomes. However, disruption of the interaction between Slac2c/MYRIP and MYO7A likely explains the defect in the transport of melanosomes into the apical processes of RPEs.

\section{Discussion}

We describe here the polka mouse line, which carries a point mutation in $M y o 7 a$ that sheds light on the function of its tail domain. The polka mutation led to aberrant splicing of Myo7a transcripts that affected its stability in the inner ear. As a consequence, the MYO7A protein was no longer expressed in the stereocilia of hair cells, leading to defects in hair bundle development. In contrast, in the retina of polka mice MYO7A protein with a truncation in the $\mathrm{FERM}_{2}$ domain were expressed at similar 


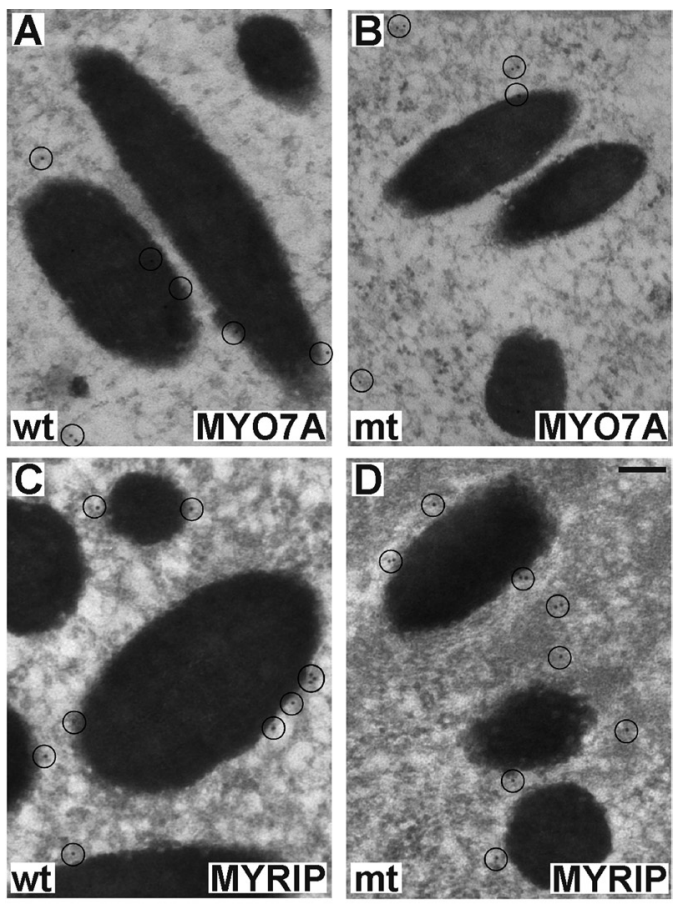

\begin{tabular}{|c|c|c|c|c|}
\hline \multirow{2}{*}{ E } & \multicolumn{2}{|l|}{ MY07A } & \multicolumn{2}{|l|}{ MYRIP } \\
\hline & d-type & polka & vild-type & polka \\
\hline $\begin{array}{l}\% \text { total gold in } \\
\text { melanosomes }\end{array}$ & 56 & 31 & 85 & 80 \\
\hline $\begin{array}{l}\text { Average gold in } \\
\text { melanosomes }\end{array}$ & $0.74 \pm 0.15$ & $0.35 \pm 0.04$ & $41.5 \pm 0.32$ & $1.3 \pm 0.1$ \\
\hline $\begin{array}{l}\text { Average gold in } \\
\text { cytoplasm basal } \\
\text { IIII average gold } \\
\text { divided by area } \\
\text { of cytoplasm }\end{array}$ & $\begin{array}{l}3.33 \pm 0.95 \\
\text { IIII } 0.11\end{array}$ & $\begin{array}{l}4.33 \pm 0.8 \\
\text { IIII } 0.16\end{array}$ & $\begin{array}{l}2.05 \pm 0.76 \\
\text { n.d. }\end{array}$ & $\begin{array}{l}2.4 \pm 0.5 \\
\text { n.d. }\end{array}$ \\
\hline $\begin{array}{l}\text { Average gold in } \\
\text { apical region }\end{array}$ & $8.6 \pm 1.5$ & $5.1 \pm 0.59$ & n.d. & n.d. \\
\hline $\begin{array}{l}\% \text { melanosomes } \\
\text { with no gold }\end{array}$ & 31 & 56 & 30 & 31 \\
\hline
\end{tabular}

Figure 9. Subcellular localization of MY07A and MYRIP in the RPE. A-D, Immunoelectron microscopy on wild-type $(\boldsymbol{A}, \boldsymbol{C})$ and mutant $(\boldsymbol{B}, \boldsymbol{D})$ RPE from retinal sections labeled with anti-MY07A and anti-MYRIP antibody and $15 \mathrm{~nm}$ protein A-gold. Gold particles have been circled. $\boldsymbol{E}$, Quantification of the immunolocalization data. Gold particles were counted in ultrathin sections. Twenty-five images were analyzed randomly for each condition and animal. Gold particles were considered to be associated with the melanosome membrane when located at a maximum of $30 \mathrm{~nm}$ from the membrane of the organelle, as described previously (Klomp et al., 2007). Values are mean \pm SEM ( $n=3$ wild-type and mutant animals). Scale bar, $100 \mathrm{~nm}$.

levels as full-length protein in wild-type mice. As a consequence, melanosome transport in RPE cells was affected. We conclude that the $\mathrm{FERM}_{2}$ domain of MYO7A is required for cargo transport into the apical processes of RPE cells. Our findings also show that a Myo $7 a$ point mutation can differentially affect gene expression in the inner ear and retina. The latter findings might explain why some mutations in MYO7A in humans only affect hearing function, while others affect both hearing and vision.

Previous studies have provided insights into the mechanisms by which mutations in Myo7a can cause disease, largely focusing on different alleles of Myo7a that affect its motor domain. While mRNA levels were not affected in mice carrying mutations in the MYO7A motor domain, protein levels were drastically reduced, leading to the suggestion that motor-domain mutations destabi- lize MYO7A (Hasson et al., 1997). In addition, some USH1 mutations in the MYO7A head domain also affect motor function of recombinant human $\mathrm{MYO} 7 \mathrm{~A}$, suggesting that defects in the interaction of MYO7A with actin also contribute to the disease (Watanabe et al., 2008).

Our findings now show that pathological changes in polka mice are caused by different mechanisms. The mutation in polka mice inactivates the exon 42 splice donor site in $M y o 7 a$ and activates a cryptic splice-donor within the intron flanked by exon 42 and 43 . Therefore a Myo7a transcript is generated with a 49 base pair intronic insert that introduces a premature stop codon. The abundance of the aberrantly spliced transcript is drastically reduced in the cochlea, likely as a consequence of nonsense-mediated mRNA decay (Isken and Maquat, 2008). MYO7A protein is also no longer detectable in stereocilia leading to defects in hair bundle development. In contrast, Myo7a transcripts escaped nonsense-mediated decay in the retina leading to the expression of a truncated MYO7A protein at similar levels as in wild-type mice. However, our findings provide evidence that the truncated protein is functionally impaired. Despite the presence of the truncated MYO7A protein in the apical processes of RPE cells, polka mice show defects in melanosome organization indistinguishable from the shaker-1 phenotype (Liu et al., 1998; Gibbs et al., 2004).

Mutant MYO7A may be misfolded or compromised in its motor function as studies with myosin $\mathrm{V}$ have shown that the tail domain regulates the function of its motor domain (Trybus et al., 1999; Homma et al., 2000; Wang et al., 2000; Thirumurugan et al., 2006). However, we think that this possibility is unlikely because mutant MYO7A is properly targeted to the apical processes of RPE, which would likely not occur with a misfolded protein or a protein without motor function. Instead, we favor the alternative hypothesis that MYO7A is unable to bind and transport cargo. Consistent with this model, the exophilin Slac2-c/MyRIP is thought to function as a linker protein between RAB27A on melanosomes and MYO7A (Fukuda and Kuroda, 2002; Kuroda and Fukuda, 2005; Klomp et al., 2007). In yeast two-hybrid and in in vitro assays Slac2-c/MyRIP can bind to the C-terminal 464 aa of MYO7A that contain the FERM 2 domain (El-Amraoui et al., 2002). Therefore, disruption of the $\mathrm{FERM}_{2}$ domain might affect interactions with melanosomes, which is consistent with our immunoelectron microscopy studies that revealed reduced association of MYO7A with melanosomes in RPEs of polka mice.

Interestingly, polka mice like previously described Myo7a alleles fail to show clear signs of retinal degeneration, highlighting the fact that melanosome localization is not critical for retinal viability (Liu et al., 1998). In fact, most, if not all mouse models of USH1 do not reproduce the retinal degeneration phenotype of USH1 patients (Williams, 2008). A recent study suggests that defective photoreceptor function might cause retinal degeneration in USH1B (Jacobson et al., 2008). The reason why genetic lesions in polka and shaker-1 alleles do not reproduce the photoreceptor degeneration found in USH1B patients remains obscure. Possibilities have been discussed previously (Liu et al., 1998; Lillo et al., 2003), but it should be noted that many mouse models of retinal degeneration, as well as other neurodegenerative disorders, mimic the pathological changes observed in humans only incompletely. Of interest, however, is the recent demonstration that mutations in the $\mathrm{FERM}_{2}$ domain of MYO7A can lead to USH1B in humans (Jaijo et al., 2006; Riazuddin et al., 
2008). Similar to the allele identified in polka mice, the FERM mutation (c.5856G $>\mathrm{A}$ ) affects the last nucleotide in exon 42 and has been predicted to influence splicing of the myo7a transcript (Jaijo et al., 2006). Based on our studies, it seems likely that such mutations in the FERM ${ }_{2}$ domain destabilized the myo7a transcript in the inner ear thereby causing defects in hair bundle development. In contrast, MYO7A protein in the retina might be functionally impaired leading to visual defects in the affected patients. As disease causing mutations have been mapped to several structural domains within the complex MYO7A tail region, it will be important to generate in the future mouse models that specifically target other structural domains besides the $\mathrm{FERM}_{2}$ domain to define their function as well as pathogenesis mechanisms that lead to visual and auditory impairment.

\section{References}

Blencowe BJ (2000) Exonic splicing enhancers: mechanism of action, diversity and role in human genetic diseases. Trends Biochem Sci 25:106-110.

Chen ZY, Hasson T, Kelley PM, Schwender BJ, Schwartz MF, Ramakrishnan M, Kimberling WJ, Mooseker MS, Corey DP (1996) Molecular cloning and domain structure of human myosin-VIIa, the gene product defective in Usher syndrome 1B. Genomics 36:440-448.

El-Amraoui A, Schonn JS, Küssel-Andermann P, Blanchard S, Desnos C, Henry JP, Wolfrum U, Darchen F, Petit C (2002) MyRIP, a novel Rab effector, enables myosin VIIa recruitment to retinal melanosomes. EMBO Rep 3:463-470.

Fariñas I, Yoshida CK, Backus C, Reichardt LF (1996) Lack of neurotrophin-3 results in death of spinal sensory neurons and premature differentiation of their precursors. Neuron 17:1065-1078.

Fukuda M, Kuroda TS (2002) Slac2-c (synaptotagmin-like protein homologue lacking C2 domains-c), a novel linker protein that interacts with Rab27, myosin Va/VIIa, and actin. J Biol Chem 277:4309643103.

Gibbs D, Kitamoto J, Williams DS (2003) Abnormal phagocytosis by retinal pigmented epithelium that lacks myosin VIIa, the Usher syndrome 1B protein. Proc Natl Acad Sci U S A 100:6481-6486.

Gibbs D, Azarian SM, Lillo C, Kitamoto J, Klomp AE, Steel KP, Libby RT, Williams DS (2004) Role of myosin VIIa and Rab27a in the motility and localization of RPE melanosomes. J Cell Sci 117:6473-6483.

Gibson F, Walsh J, Mburu P, Varela A, Brown KA, Antonio M, Beisel KW, Steel KP, Brown SD (1995) A type VII myosin encoded by the mouse deafness gene shaker-1. Nature 374:62-64.

Hasson T, Heintzelman MB, Santos-Sacchi J, Corey DP, Mooseker MS (1995) Expression in cochlea and retina of myosin VIIa, the gene product defective in Usher syndrome type 1B. Proc Natl Acad Sci U S A 92:9815-9819.

Hasson T, Walsh J, Cable J, Mooseker MS, Brown SD, Steel KP (1997) Effects of shaker-1 mutations on myosin-VIIa protein and mRNA expression. Cell Motil Cytoskeleton 37:127-138.

Holme RH, Steel KP (2002) Stereocilia defects in waltzer (Cdh23), shaker1 (Myo7a) and double waltzer/shaker1 mutant mice. Hear Res 169:13-23.

Homma K, Saito J, Ikebe R, Ikebe M (2000) $\mathrm{Ca}(2+)$-dependent regulation of the motor activity of myosin V. J Biol Chem 275:34766-34771.

Isken O, Maquat LE (2008) The multiple lives of NMD factors: balancing roles in gene and genome regulation. Nat Rev Genet 9:699-712.

Jacobson SG, Cideciyan AV, Aleman TS, Sumaroka A, Roman AJ, Gardner LM, Prosser HM, Mishra M, Bech-Hansen NT, Herrera W, Schwartz SB, Liu XZ, Kimberling WJ, Steel KP, Williams DS (2008) Usher syndromes due to MYO7A, PCDH15, USH2A or GPR98 mutations share retinal disease mechanism. Hum Mol Genet 17:2405-2415.

Jaijo T, Aller E, Oltra S, Beneyto M, Najera C, Ayuso C, Baiget M, Carballo M, Antinolo G, Valverde D, Moreno F, Vilela C, Perez-Garrigues H, Navea A, Millan JM (2006) Mutation profile of the MYO7A gene in Spanish patients with Usher syndrome type I. Hum Mutat 27:290-291.

Klomp AE, Teofilo K, Legacki E, Williams DS (2007) Analysis of the linkage of MYRIP and MYO7A to melanosomes by RAB27A in retinal pigment epithelial cells. Cell Motil Cytoskeleton 64:474-487.

Krawczak M, Thomas NS, Hundrieser B, Mort M, Wittig M, Hampe J,
Cooper DN (2007) Single base-pair substitutions in exon-intron junctions of human genes: nature, distribution, and consequences for mRNA splicing. Hum Mutat 28:150-158.

Kros CJ, Marcotti W, van Netten SM, Self TJ, Libby RT, Brown SD, Richardson GP, Steel KP (2002) Reduced climbing and increased slipping adaptation in cochlear hair cells of mice with Myo7a mutations. Nat Neurosci 5:41-47.

Kuroda TS, Fukuda M (2005) Functional analysis of Slac2-c/MyRIP as a linker protein between melanosomes and myosin VIIa. J Biol Chem 280:28015-28022.

Lillo C, Kitamoto J, Liu X, Quint E, Steel KP, Williams DS (2003) Mouse models for Usher syndrome 1B. Adv Exp Med Biol 533:143-150.

Liu X, Ondek B, Williams DS (1998) Mutant myosin VIIa causes defective melanosome distribution in the RPE of shaker-1 mice. Nat Genet 19:117-118.

Liu X, Udovichenko IP, Brown SD, Steel KP, Williams DS (1999) Myosin VIIa participates in opsin transport through the photoreceptor cilium. J Neurosci 19:6267-6274.

Liu XZ, Walsh J, Mburu P, Kendrick-Jones J, Cope MJ, Steel KP, Brown SD (1997) Mutations in the myosin VIIA gene cause non-syndromic recessive deafness. Nat Genet 16:188-190.

Manly KF, Cudmore RH Jr, Meer JM (2001) Map Manager QTX, crossplatform software for genetic mapping. Mamm Genome 12:930-932.

Mburu P, Liu XZ, Walsh J, Saw D Jr, Cope MJ, Gibson F, Kendrick-Jones J, Steel KP, Brown SD (1997) Mutation analysis of the mouse myosin VIIA deafness gene. Genes Funct 1:191-203.

Müller U, Wang D, Denda S, Meneses JJ, Pedersen RA, Reichardt LF (1997) Integrin alpha8betal is critically important for epithelial-mesenchymal interactions during kidney morphogenesis. Cell 88:603-613.

Reijmers LG, Coats JK, Pletcher MT, Wiltshire T, Tarantino LM, Mayford M (2006) A mutant mouse with a highly specific contextual fear-conditioning deficit found in an N-ethyl-N-nitrosourea (ENU) mutagenesis screen. Learn Mem 13:143-149.

Riazuddin S, Nazli S, Ahmed ZM, Yang Y, Zulfiqar F, Shaikh RS, Zafar AU, Khan SN, Sabar F, Javid FT, Wilcox ER, Tsilou E, Boger ET, Sellers JR, Belyantseva IA, Riazuddin S, Friedman TB (2008) Mutation spectrum of MYO7A and evaluation of a novel nonsyndromic deafness DFNB2 allele with residual function. Hum Mutat 29:502-511.

Rinchik EM, Carpenter DA (1999) N-Ethyl-N-nitrosourea mutagenesis of a 6- to 11-cM subregion of the Fah-Hbb interval of mouse chromosome 7: completed testing of 4557 gametes and deletion mapping and complementation analysis of 31 mutations. Genetics 152:373-383.

Rinchik EM, Carpenter DA, Selby PB (1990) A strategy for fine-structure functional analysis of a 6- to 11-centimorgan region of mouse chromosome 7 by high-efficiency mutagenesis. Proc Natl Acad Sci U S A 87:896-900.

Schwander M, Sczaniecka A, Grillet N, Bailey JS, Avenarius M, Najmabadi H, Steffy BM, Federe GC, Lagler EA, Banan R, Hice R, Grabowski-Boase L, Keithley EM, Ryan AF, Housley GD, Wiltshire T, Smith RJ, Tarantino LM, Müller U (2007) A forward genetics screen in mice identifies recessive deafness traits and reveals that pejvakin is essential for outer hair cell function. J Neurosci 27:2163-2175.

Self T, Mahony M, Fleming J, Walsh J, Brown SD, Steel KP (1998) Shaker-1 mutations reveal roles for myosin VIIA in both development and function of cochlear hair cells. Development 125:557-566.

Senapathy P, Shapiro MB, Harris NL (1990) Splice junctions, branch point sites, and exons: sequence statistics, identification, and applications to genome project. Methods Enzymol 183:252-278.

Senften M, Schwander M, Kazmierczak P, Lillo C, Shin JB, Hasson T, Géléoc GS, Gillespie PG, Williams D, Holt JR, Müller U (2006) Physical and functional interaction between protocadherin 15 and myosin VIIa in mechanosensory hair cells. J Neurosci 26:2060-2071.

Soni LE, Warren CM, Bucci C, Orten DJ, Hasson T (2005) The unconventional myosin-VIIa associates with lysosomes. Cell Motil Cytoskeleton 62:13-26.

Thirumurugan K, Sakamoto T, Hammer JA 3rd, Sellers JR, Knight PJ (2006) The cargo-binding domain regulates structure and activity of myosin 5 . Nature 442:212-215.

Trybus KM, Krementsova E, Freyzon Y (1999) Kinetic characterization of a monomeric unconventional myosin V construct. J Biol Chem 274: 27448-27456. 
Wang F, Chen L, Arcucci O, Harvey EV, Bowers B, Xu Y, Hammer JA 3rd, Sellers JR (2000) Effect of ADP and ionic strength on the kinetic and motile properties of recombinant mouse myosin V. J Biol Chem 275:4329-4335.

Watanabe S, Umeki N, Ikebe R, Ikebe M (2008) Impacts of Usher syndrome type IB mutations on human myosin VIIa motor function. Biochemistry 47:9505-9513.

Weil D, Blanchard S, Kaplan J, Guilford P, Gibson F, Walsh J, Mburu P, Varela A, Levilliers J, Weston MD, Kelley PM, Kimberling WJ, Wagenaar M, Levi-Acobas F, Larget-Piet D, Munnich A, Steel KP, Brown SDM, Petit C (1995) Defective myosin VIIA gene responsible for Usher syndrome type 1B. Nature 374:60-61.
Williams DS (2008) Usher syndrome: animal models, retinal function of Usher proteins, and prospects for gene therapy. Vision Res 48:433-441.

Wiltshire T, Pletcher MT, Batalov S, Barnes SW, Tarantino LM, Cooke MP, Wu H, Smylie K, Santrosyan A, Copeland NG, Jenkins NA, Kalush F, Mural RJ, Glynne RJ, Kay SA, Adams MD, Fletcher CF (2003) Genomewide single-nucleotide polymorphism analysis defines haplotype patterns in mouse. Proc Natl Acad Sci U S A 100:3380-3385.

Wolfrum U, Liu X, Schmitt A, Udovichenko IP, Williams DS (1998) Myosin VIIa as a common component of cilia and microvilli. Cell Motil Cytoskeleton 40:261-271.

Zheng QY, Johnson KR, Erway LC (1999) Assessment of hearing in 80 inbred strains of mice by ABR threshold analyses. Hear Res 130:94-107. 